\title{
Codesigning biodiversity-based agrosystems promotes alternatives to mycorrhizal inoculants
}

\author{
Marie Chave ${ }^{1}(1) \cdot$ Valérie Angeon $^{2} \cdot$ Raphaël Paut $^{1,2} \cdot$ Robin Collombet $^{1,2} \cdot$ Marc Tchamitchian $^{2}$
}

Accepted: 10 September 2019 / Published online: 24 October 2019

(C) The Author(s) 2019

\begin{abstract}
Facing the challenge of the ecological transition of agriculture, biodiversity opens new avenues to enhance ecological interactions and reduce chemical input dependency. Designing biodiversity-based agrosystems requires an agroecological approach that combines key principles: exploring a wide range of concepts and solutions, adopting systemic reasoning, implementing a sitespecific approach, developing an action-oriented process, and maintaining a continuous improvement dynamic. This type of approach has never been developed to harness mycorrhizal fungi, which are key components of soil biodiversity, because their beneficial action on crops depends on complex and underexploited ecological interactions. At present, mycorrhizae are mainly used through industrial inoculants that fit within the productionist paradigm. To shift toward agroecological approaches, we implemented a methodological framework conceived to better address the design of mycorrhiza-friendly cropping systems by sharing knowledge with farmers in four different study areas (Provence, French Guiana, Guadeloupe, and Martinique). This framework includes participative workshops, a board game, and prospective exercises to collect farmers' proposals and the factors that prevent from implementing mycorrhiza-friendly cropping systems. We showed that $90 \%$ of the farmers proposed alternatives to industrial inoculants, $50 \%$ of them adopted systemic reasoning by combining these alternative proposals. Most farmers understood that they were all potential "mycorrhizae producers". We showed, for the first time through on-farm experiments that valorization of indigenous mycorrhizal fungi strains using a donor plant is an effective practice to increase root colonization before planting (up to a frequency of $95 \%$ and an intensity of 32\%). Considering the increasing supply of mycorrhizal inoculants and despite the uncertainty of related knowledge, we codesigned innovative practices. Learning communities (technical advisors, high school teachers, etc.) assumed responsibility for continuous improvement in knowledge and practices. Finally, beyond the issue of mycorrhizae, we showed that an agroecological approach could bring stakeholders one step further into the design of biodiversity-based agrosystems.
\end{abstract}

Keywords Agroecology $\cdot$ Innovation $\cdot$ Participatory design $\cdot$ Soil biodiversity $\cdot$ Arbuscular mycorrhizal fungi

\section{Introduction}

Agroecological transition addresses the paramount challenge of feeding a growing population with scarce resources while preserving the environment (Wezel and Soldat 2009). Facing the limits of the productionist paradigm based on the artificialization of agrosystems (anthropogenic inputs, monocropping, heavy mechanization), several approaches to

Marie Chave

marie.chave@inra.fr

1 ASTRO (UR 1321), INRA, 97170 Petit-Bourg, Guadeloupe, France

2 ECODEVELOPPEMENT (UR 767), INRA, 84000 Avignon, France the development of sustainable agriculture coexist. They can be described through the efficiency-substitution-redesign framework (Hill and MacRae 1995), which leads to the design of agrosystems based on the valorization of biodiversity. A review of the prolific literature on agroecology shows that the transition toward "biodiversity-based agrosystems" (Duru et al. 2015) requires an agroecological approach. Such an approach is based on five unavoidable principles (Martin 2015; Méndez et al. 2013).

First, biodiversity-based agrosystems call for an exploration of a wide range of concepts and solutions (Salembier et al. 2018). Overlooked until recently, ecological interactions offer new lines of action for crop health and productivity management. Because "one size fits all" solutions are no longer suitable to address the diverse contexts faced by farmers, a range 
of solutions must be proposed. Second, systemic reasoning allows users to grasp the complexity of biodiversity-based agrosystems and manage multiobjective and embedded solutions. Indeed, ecological interactions (particularly those in soil (Bender et al. 2016)) involve communities belonging to different species that interact though poorly understood and complex processes that evolve over several years and work across multiple scales. Thus, implementing biodiversity-based agrosystems requires holistic and strategic thinking. Third, to adapt agricultural practices to local pedoclimatic and sociotechnical contexts, site-specific implementations must be provided (Duru et al. 2015). From a variety of solutions, farmers must be given the possibility to establish their own trade-offs, relying on their own knowledge, scientific knowledge, and available products and technologies (Meynard et al. 2012). Such a conception admits agroecology as intrinsically inclusive because considering stakeholders' points of view, their empirical knowledge and their expertise imply a major recognition of their place and role in decision-making processes (Wezel and Soldat 2009). Fourth, an action-oriented approach means that stakeholders test and experiment with solutions (trial and error process) (Méndez et al. 2013). By building knowledge in practice, through experience, stakeholders take ownership of new solutions and develop pragmatic actions. In that sense, agroecology aims at impelling transformative change. Fifth, stakeholders must place themselves in a continuous improvement dynamic, involving the permanent evaluation and tuning of practices (Martin 2015; Duru et al. 2015). This requirement can lead to the empowerment of actors in designing and monitoring of biodiversity-based agrosystems.

In the design of biodiversity-based agrosystems, arbuscular mycorrhizal fungi, key components of soil biodiversity, offer an underexploited potential (Bender et al. 2016). Present in most soils worldwide, arbuscular mycorrhizal fungi colonize more than $80 \%$ of plant species (including most crops) and furnish a wide diversity of ecosystem services that enhance crop health and productivity (Smith and Read 2008). To develop and reproduce, arbuscular mycorrhizal fungi must establish a symbiosis with a host plant, whether from a spore, mycorrhizal root fragment, or mycelium. After symbiosis is established, the development of a dense mycelium increases the surface area used for soil exploration by crop roots and favors nutrient absorption. In return, plants provide carbon resources to the fungi. The fungal mycelia can colonize the roots of several plants of different species, linking them together and forming a common mycorrhizal network to exchange carbon, nutrients, and other elements. Although not all the interactions in this process are completely understood, recent studies have shown that interplant communications via mycorrhizal networks also contribute to plant protection (Johnson and Gilbert 2015).

The current solutions available for farmers to harness mycorrhizae are composed of standard propagules (spore, mycorrhizal root fragment, or mycelium) produced industrially from a few selected strains (Hart et al. 2018). This strategy complies with the dominant sociotechnical system relying on the productionist paradigm based on anthropogenic input efficiency or substitution. Industrial strains are available for inoculation in nurseries and in fields with different packaging (liquid, bags, seed coating, etc.). In France, for example, at least thirteen products including mycorrhizae are registered and commercialized as fertilizing material and culture media (articles L.255-1 to L.255-11 of the French rural and fishery code). They all contain the same strain of mycorrhizal fungus (Rhizoglomus irregulare DAOM 181602/197198), selected because of its high reproductive capacity. However, inoculation success with such selected strains in farming conditions is not guaranteed. Indeed, competition for ecological niches related to the capacity of a given species to grow under certain soil conditions and interspecific competition with indigenous strains may prevent the inoculated strain from bonding with the target plant or conversely may represent an invasive risk. Moreover, commercial mycorrhiza-based products are sold with little explanation of the agricultural practices that are crucial for their establishment (e.g., light tillage, crop rotation, limited fertilization, and pesticide applications (Jansa et al. 2006)). Thus, poor timing of inoculation and inappropriate management practices may compromise the success of symbiosis establishment and mycorrhizal network development, respectively (Verbruggen et al. 2013; Hart et al. 2018).

Whilst enhancing colonization by indigenous arbuscular mycorrhizal fungi seems to be a promising alternative practice (Pellegrino et al. 2011), yield benefits provided by such a crop management are controversial. Some authors argue that the literature presents an overoptimistic vision of the impacts of arbuscular mycorrhizal fungi on yields (Ryan and Graham 2018; Ryan et al. 2019). Others reply that limiting the analysis to yields is restrictive in view of the many services that arbuscular mycorrhizal fungi can provide to contribute to the sustainability of agrosystems (Rillig et al. 2018). Nevertheless, most authors agree that evaluating the impacts of arbuscular mycorrhizal fungi on yields in field experiments is very challenging especially because of the difficulty of producing noncolonized control plants.

Facing a growing interest in mycorrhizae and the incompleteness of scientific and practical knowledge, we elaborated a methodological framework to codesign mycorrhiza-friendly agrosystems (Chave and Angeon 2018). This framework, called MYMYX ("Mimic mycorrhizal networks") is a learning tool that supports a participatory approach to favor the emergence of biodiversity-based agrosystems. It helps users share knowledge about ecological processes and allows farmers to use, hybridize, and implement relevant knowledge and necessary skills, in keeping with the "more knowledge per hectare" call (Buckwell et al. 2014). In this paper, we present and discuss the successes and limitations of MYMYX with 
respect to the five key principles of design for biodiversitybased agrosystems previously presented.

\section{Material and methods}

\subsection{Study areas and sampling}

In the context of an increasing number of offers for commercial mycorrhiza-based products (Hart et al. 2018), an explicit demand for farmer training on mycorrhizae was formulated to INRA (French National Institute for Agricultural Research) by the agricultural extension services of different areas in the tropics: FREDON (Regional Federation for Defense against Bioaggressors) in Martinique and the Chambers of Agriculture in French Guiana and Guadeloupe. To extend the study to a different sociotechnical context (metropolitan, non-isolated, temperate climate), we involved another group of farmers in Provence. In these four contrasting study areas, 50 farmers were involved: 19 farmers in Martinique, 14 farmers in French Guiana, seven farmers in Guadeloupe, and ten farmers in Provence. Farmers were selected by institutional actors as members of their proximity networks sensitized to agroecological issues. Thus, the selected farmers are not representative of farmer diversity in the study areas. Most farmers involved were professional market gardeners, but production orientation and farm size differed, both in each location and among different locations. MYMYX was conceived to address these development issues.

\subsection{MYMYX: a methodological framework for harnessing mycorrhizae with farmers}

Inspired by the innovative design $\mathrm{KCP} \circledast$ methodology (Knowledge Concept Proposition, Le Masson et al. 2009), MYMYX (Chave and Angeon 2018) gathers researchers and farmers in a three-step design strategy aiming to explore how to enhance and benefit from mycorrhizae: a first sharingknowledge workshop, on-farm surveys, and a final prospective workshop.

The approach started with a half-day collaborative workshop to foster knowledge exchanges among participants and to allow them to internalize the concepts of mycorrhization. A learning support tool — a board game (Fig. 1) — served as a boundary object. A boundary object is defined by Star and Griesemer (1989) as "an entity shared by several different communities but viewed or used differently by each of them, being both plastic enough to adapt to local needs and the constraints of the several parties employing them, yet robust enough to maintain a common identity across sites." Four main questions were addressed: (i) What are the benefits of mycorrhizae? (ii) How can a mycorrhizal network be established? (iii) How can density be increased in the mycorrhizal network once it is created? and (iv) Which practices are

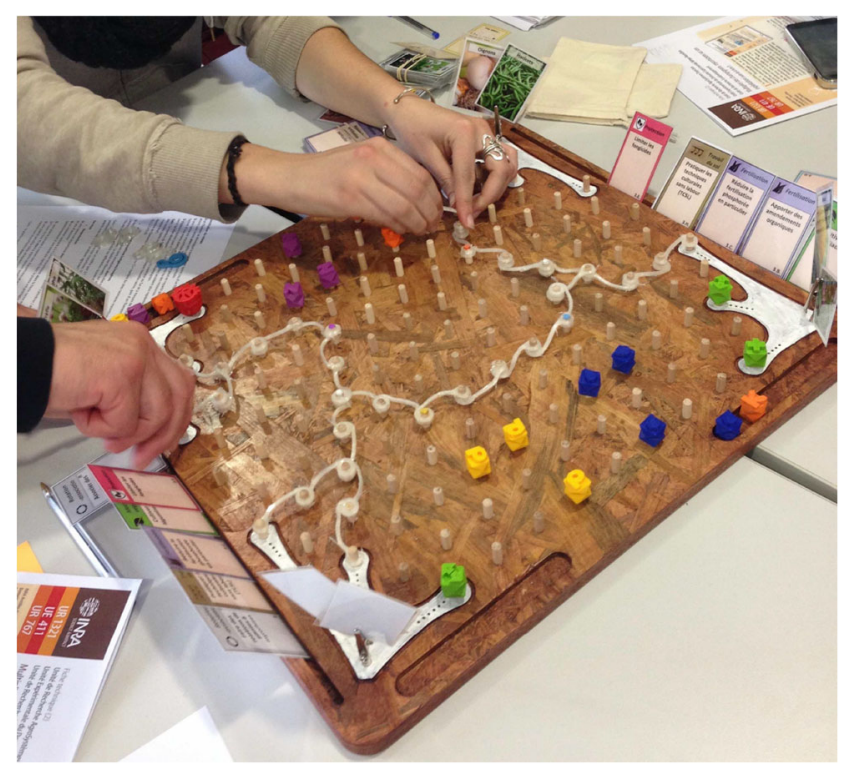

Fig. 1 The board game used during the first MYMYX workshop. The game board provides support for moving mobile elements representing mycorrhizal filaments (in white), allowing the players to build a network between the plant roots, which are symbolized by white markings at the four corners of the board. Players propose agricultural practices (with the cards on the sides of the board) to earn filaments, which allow them to link the roots with the nutritive resources (i.e., phosphorus, yellow $\mathrm{P}$ pieces, or water, blue pieces)

farmers willing (or not willing) to implement to foster mycorrhizae? To answer these three last questions, farmers use cards, proposed by researchers, within six categories of agricultural practice (favorable and unfavorable): choice of target plant (e.g., alliaceous, brassicaceous), tillage (e.g., light tillage, plowing), inputs (e.g., compost, fertilizer), crop rotation (e.g., intercropping, monocropping), introduction of propagules (e.g., on-farm production, standard propagules), crop protection (e.g., limiting fungicide, solarization). Farmers were invited to formulate and write new agricultural practices on blank cards to address the questions throughout the workshop. All these proposals were collected in a database. Each card played was discussed among the farmers in groups. Following this phase, the farmers collectively built a cropping strategy combining several practice cards to develop a mycorrhizal network among the crops using the board game (Fig. 1).

The second step was an on-farm survey based on semistructured questionnaires carried out 2 months later with all the farmers who attended the first workshop. The objectives were to assess knowledge retention, to continue proposal collection, and to identify the different types of constraints to mycorrhizal mobilization. The fifteen questions used to identify constraints focused on five aspects: (i) Farmers' level of environmental concern. (ii) Knowledge acquired about mycorrhizae. (iii) Agronomic constraints and implications of mycorrhizal mobilization in terms of inputs, soil tillage, crop rotations, etc. (iv) Economic implications for their farms. (v) Level of farmer experience with mycorrhizae (either their own 
experience or information from their sociotechnical networks). To quantify the strength of the constraints communicated by the farmers, we attributed a qualitative score from zero to three to every answer related to constraints in the interviews. This score was assigned with a unified grid tailored to each question, where zero was no constraint identified, one was expression of a non-limiting constraint, two was a somewhat limiting constraint, and three was expression of a blocking constraint. We applied a killer criterion for all categories of constraints, except for the experience constraint. When a score for one of the answers was "three," the constraint was rated with a three. The experience constraint was not treated as a "killer criterion" because it would not make sense to do so since not having feedback is not a blocking constraint. We averaged the scores from two to four questions per constraint category to derive a unified score. Three specific questions also allowed quantification of technical constraints, according to the farmers and with the same scoring methodology, over three key practices: tillage reduction, input reduction, and crop rotation. The database of proposals was enriched through this stage.

The third step was the second half-day collaborative workshop held to combine the proposals into a design strategy. This workshop was organized in two phases: (i) a presentation of the results of the survey and a debate on recent experimentation, and (ii) a prospective evaluation to orient collective creativity for the future for mycorrhizal enhancement. All steps were implemented in every study area over a period of 3 to 4 months.

\subsection{Inventory and classification of farmers' proposals}

In our study, a proposal is the formulation by farmers of an idea, an opinion, or a point of view that is discussed within a group. Proposals can be technical or organizational at the individual or collective scale. The proposal can fall within different concepts and can lead to concrete solutions. Within this set of proposals, we classified individual agronomic proposals though underlying biological processes leading to a hierarchical concept tree divided into three concepts (Chave et al. 2014). The first concept, the introduction of propagules, refers to the addition of arbuscular mycorrhizal fungi propagules to the agrosystem. It includes the use of commercial standard strains or indigenous strains produced on-farm or locally. The second concept, connection of mycorrhizal fungi with plants, refers to the establishment of symbiosis between mycorrhizal fungi and target crops. This symbiosis can be achieved through a "network effect" by transmitting the fungi from a mycorrhiza-friendly host plant to a target plant via intercropping or rotation. The third concept, densification of mycorrhizal networks, refers to the limitation of the damage produced by agricultural practices on established mycorrhizal networks, including the reduction of soil disturbance and the reduction of chemical inputs (pesticides, chemical fertilizers) (Verbruggen et al. 2013).

We distinguished proposals specifically targeted toward harnessing mycorrhizae from multiobjective ones (i.e., proposals that include additional objectives rather than being strictly focused on mycorrhizal processes such as inoculation with propagules).

\subsection{Statistical analysis}

To identify the relationship between the constraints expressed by farmers and the proposals they made, we carried out a multiple factorial analysis (MFA), followed by a hierarchical cluster analysis on principal components (HCPC). The explanatory variables used were the five constraint scores and the three concepts mobilized in farmers' proposals as dichotomous variables ( 0 : concept not expressed, 1 : concept expressed). We also included the farm location and certification as supplementary qualitative variables to assist in the interpretation of components and clusters. Hierarchical clustering analysis was performed on the first two components identified with the multifactorial analysis. We tested the ingroup distribution of quantitative variables (Kuiper's $V$ test) or qualitative variables (hypergeometric test) against the whole sample for each group in order to identify discriminating variables. Significantly different variables $(P<0.05)$ were considered characteristic of the group.

To further explore the relationships between the farmers' locations and the types of practice they proposed, we carried out a bipartite network analysis (location and associated practices) using the igraph $\mathrm{R}$ package. We used the FruchtermanReingold algorithm to build the network, with connection weight being proportional to the number of proposals generated per location.

We compared the average constraint scores between each location with the Kruskal-Wallis test, followed (when significant, $P<0.05$ ) by Dunn's test with the Bonferroni adjustment. We compared constraint scores between two pools of farmers (combination of clusters) with the Wilcoxon test (with $\alpha=$ 0.05). We made pairwise comparisons of the five constraint scores over the whole sample with Dunn's test with the Bonferroni adjustment. All statistical analyses were conducted using R (v3.4.4) (R Core Team 2018) with the packages FactoMineR and FSA.

\section{Results and discussion}

Due to the differing contexts and sociotechnical networks, the farms' characteristics varied among study areas. Nonetheless, all farmers were involved in a trajectory of ecologization of their practices, although the proportion of organic-certified farmers varied from one location to another: Provence, $90 \%$; 
French Guiana, 57\%; Martinique, 26\%; and Guadeloupe, $0 \%$. In Martinique, the group was composed of farmers belonging to the "agroecological network" driven by the FREDON. Market gardening was the primary production system (average farm size of $5.5 \mathrm{ha}$ ), with fruit production being a secondary production system for five farms. In French Guiana, farmers were accustomed to work in collaboration with the Chamber of Agriculture through the EcoPhyto (governmental initiative to reduce pesticides) program. Ten farmers had fruit production, and seven farmers also had livestock operations, which explain the higher average farm size ( $52 \mathrm{ha}$ ) in this sample. Guadeloupe farmers were all sugarcane growers belonging to the Chamber of Agriculture "Dephy Ferme Guadeloupe" network, with market gardening as a secondary production system (average farm size, $9.3 \mathrm{ha}$ ). In Provence, farmers came from several channels ("Dephy Ferme Provence," the Agricultural Chamber, "Maraîchage sur sol vivant," GRAB (Research Group on Organic Agriculture), and CIVAM (Center for initiatives to promote agriculture and the rural environment)). Market gardening was the primary production system (average farm size, $5.1 \mathrm{ha}$ ), with fruit production being a secondary production system for two farms.

Based on the implementation of MYMYX with these farmers, we analyze and discuss its ability to address the five key principles of the design of biodiversity-based agrosystems: exploring a wide range of concepts and solutions, adopting systemic reasoning, implementing a sitespecific approach, developing an action-oriented process, and maintaining a continuous improvement dynamic (Fig. 2). The following sections consider these five key principles one by one.

\subsection{Exploring a wide range of proposals and concepts to produce solutions to harness mycorrhizae}

The farmers in each study area generated a wide range of proposals. Overall, they made 154 agronomic proposals aiming at harnessing mycorrhizae. Figure 3 presents the hierarchical concept tree resulting from the farmers' proposals. It is structured in three levels: concepts, subconcepts, and practices. The concepts explored were the introduction of propagules $(\mathrm{C} 1)$, the connection of mycorrhizal fungi with plants (C2), and the densification of mycorrhizal networks (C3). Proposals could divide each concept into several subconcepts based on the agronomic principles involved. The $\mathrm{C} 1 \mathrm{concept}$ was divided between the use of standard commercial mycorrhizal strains and the use of indigenous (naturally occurring) strains. The $\mathrm{C} 2$ concept was divided between spatial and temporal management of the connections between mycorrhizal fungi and host plants. Spatial management relies on a direct mycorrhizal network effect between a host and the target crop grown together in the same plot (intercropping). Temporal management exploits the availability of living mycorrhizal networks in the previous host plants' remaining root systems for relay crop mycorrhization. The C3 concept relies on two complementary subconcepts, avoiding mycorrhizal network disruption (by reducing soil disturbance) and maintaining the favorable chemical properties of soils (by limiting excessive nutrient availability and harmful pesticides). These different subconcepts are illustrated by solutions that are effective agronomic practices that can be implemented by farmers in their fields (e.g., on-farm propagule production, green manure, agroforestry, mulch).

The vast majority of proposals involved concepts $\mathrm{C} 2$ (48\%) and $\mathrm{C} 3(36 \%)$; introducing propagules $(\mathrm{C} 1)$ represented only $16 \%$ of proposals. Surprisingly, the introduction of standard strain-based propagules, which is the current available technology (Hart et al. 2018), was proposed by $6 \%$ of the farmers and represented only $2 \%$ of the proposals. Rather, farmers proposed multiplying the indigenous propagules $(14 \%)$ as an alternative to buying standard strains. However, on-farm indigenous mycorrhizae production is labor-intensive, uncertain, and costly (Douds et al. 2005). Farmers in Martinique made an innovative proposal to address these limitations. It consisted of creating a collective platform including a few farmers with similar pedoclimatic conditions to produce indigenous mycorrhizae together.

Most agronomic practices can be classified as multiobjective (Fig. 3). For example, intercropping legumes with a main crop is a practice used to enhance soil fertility via biological nitrogen fixation (Sinoquet and Cruz 1995). Intercropping with an alliaceous plant such as onion, on the other hand, favors mycorrhizae and onion is also used as a sanitizing crop against Ralstonia solanacearum, a major soilborne pathogen of vegetables in the French West Indies (Chave et al. 2014). One-fifth of the practices were specifically targeted to the development of mycorrhizae. For example, inducing water stress at a convenient time during the agricultural production cycle illustrates farmers' internalization of the underlying concepts of plant-mycorrhizal symbiosis, as stressing plants is not an idea likely to spontaneously occur to farmers (Augé 2001). MYMYX raised farmers' awareness of the potential of known and novel practices to harness mycorrhizae. Some of the farmers integrated mycorrhizal potential as a criterion for designing their cropping system.

Interestingly, the repartition of proposals shows that farmers had much greater interest in subconcepts that diverged from the productionist paradigm (i.e., association and rotation vs. monocropping). This preference could be explained by two hypotheses. First, knowledge sharing through MYMYX during the first workshop led farmers to understand that mycorrhization was a multifactorial issue requiring new methods outside of the productionist paradigm. Second, all farmers were already acquainted with the "ecologically integrated paradigm" due to their participation in input reduction networks. 


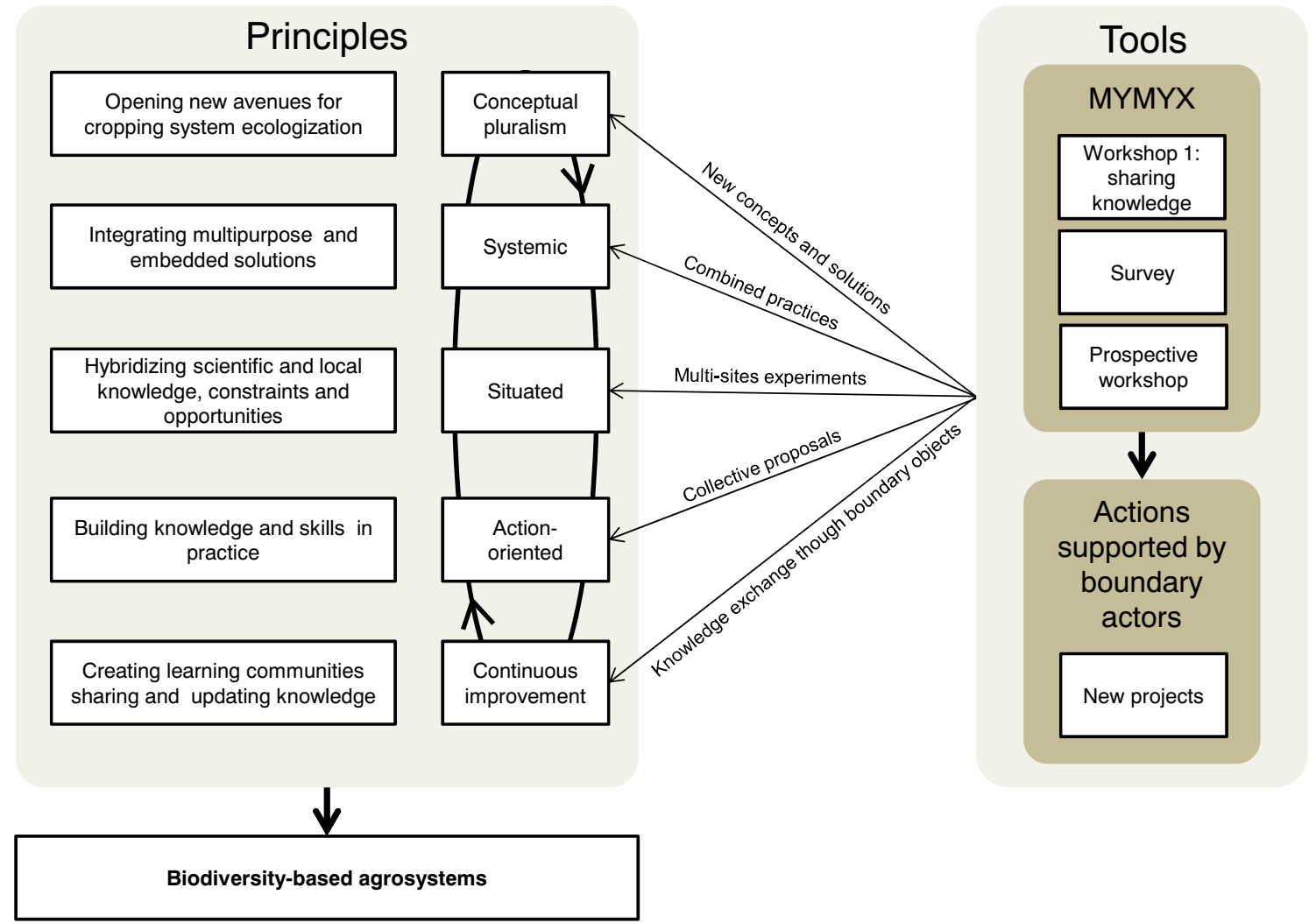

Fig. 2 Contribution of MYMYX to the design of biodiversity-based agrosystems. MYMYX followed by actions supported by boundary actors are consistent with the key interrelated principles of an agroecological approach: exploring a wide range of concepts and solutions, adopting systemic reasoning, implementing a site-specific approach, developing an action-oriented process, and maintaining a continuous improvement dynamic (based on Méndez et al. 2013; Duru et al. 2015; Martin 2015)
Although MYMYX has a limited time frame (two half-day workshops), it opened a wide if not exhaustive range of solutions to harnessing mycorrhizae, leading farmers to understand that they were all potentially "mycorrhizae producers." This diversity of solutions must be integrated into systemic reasoning.

\subsection{Adopting systemic reasoning}

Among the 50 farmers participating in MYMYX, 47 made from one to six proposals (while three made none) using various combinations of the three concepts. Constraint scores (economic, agronomic, knowledge, environment, experience) also varied between farmers, from an average of 0.1 to 3 . The multifactorial analysis distinguished farmers based on the combinations of concepts they used as well as on the intensity of the constraints they expressed for each category. It provided a two-dimensional model explaining $52 \%$ of the variance. The first dimension of the multifactorial analysis was negatively correlated with proposals related to concepts $\mathrm{C} 1$ and $\mathrm{C} 3$ and positively correlated with constraint scores for economy, environment, agronomy, and knowledge. The second dimension was negatively correlated with the constraint score for experience and with proposals related to concept $\mathrm{C} 2$ (mycorrhizal fungi-plant connection). The experience constraint was clearly uncorrelated with the other constraints and allowed a distinction among the farmers in the second dimension. The hierarchical clustering yielded five clusters for a maximal inertia gain (Table 1). We further distinguished two pools of farmers by regrouping clusters to combine farmers with similar characteristics in proposition dynamism and constraint level: a "proactive pool" and a "reserved pool."

The "proactive pool" included 23 farmers from clusters 1 and 2. Overall, these farmers made $76 \%$ of all proposals, with an average of 4.9 proposals per farmer. Farmers from cluster 1 had significantly lower economic constraint score, while cluster 2 farmers had significantly lower environmental constraint score (Table 1). In addition, technical constraints (for diversifying crop rotation and reducing inputs and tillage) were significantly lower (Mann-Whitney, $P=0.03$ ) in the proactive pool. All farmers from the proactive pool generated combined solutions involving both concepts $\mathrm{C} 2$ and $\mathrm{C} 3$ to favor mycorrhizal fungi-plant connection and mycorrhizal network development through various practices. This result indicates that they understand that agronomic management is an effective way of harnessing mycorrhizae. In addition, 17 proposals from the proactive pool hybridized two concepts into one practice. This ability of farmers from the proactive pool to 


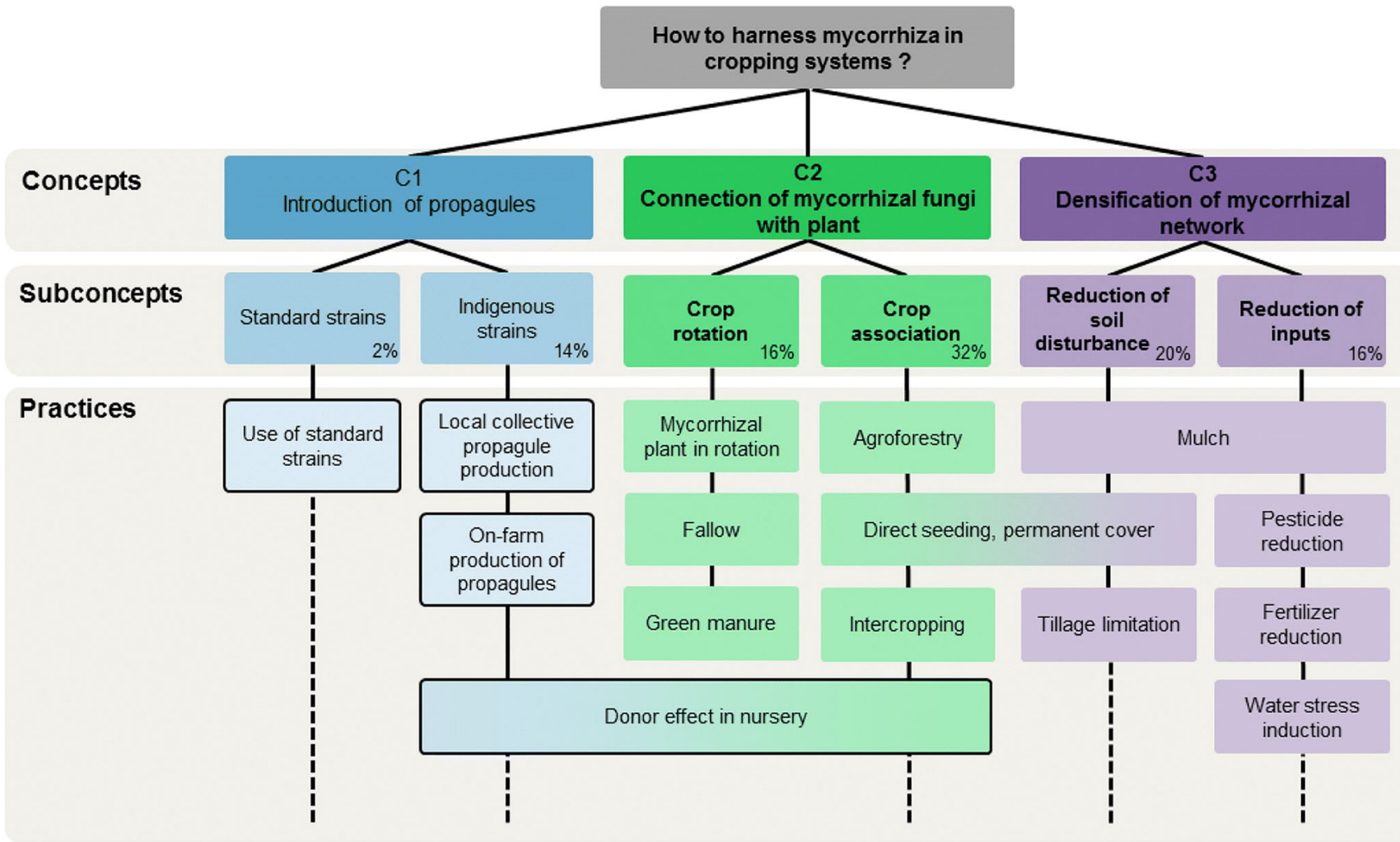

Fig. 3 Diversity of concepts and practices mobilized by farmers to harness mycorrhiza in cropping systems. Concepts are based on the steps of the mycorrhization process and are divided into subconcepts based on agronomic principles. Practices group together several proposals (from the farmers' 154 proposals) related to the subconcepts.

combine practices via systemic reasoning places them as part of a redesign approach (Hill and MacRae 1995). For example, proposals centered on the use of living mulch and direct seeding involve systemic changes at the farm level (new machinery, new rotations, new crops). They aim to decrease soil disturbance and chemical inputs (C3) while preserving the mycorrhiza-friendly root system of the cover crop (often fabaceous) for faster and stronger development of mycorrhizal fungi in the main crop (C2). Only ten farmers from cluster 1 used the full range of concepts by including the introduction of propagules $(\mathrm{C} 1)$ in their proposals. One-third of these $\mathrm{C} 1$ proposals concerned a device aimed at directly transferring indigenous mycorrhizae from a donor crop intercropped with a target crop in the nursery $(\mathrm{C} 1)$ through the donor effect $(\mathrm{C} 2)$ as demonstrated in vitro by Voets et al. (2009). This innovative design illustrates the potential of the MYMYX approach to lead farmers to hybridize concepts to produce new proposals for subsequent evaluation.

The "reserved pool" included the remaining 27 farmers from clusters 3,4 , and 5 , who, on average, made only 1.3 proposals per farmer. Average constraint scores were significantly higher for only cluster 5 (all constraints). The farmers in this pool were mainly distinguished by their lower propensity for making proposals and combining concepts. The farmers
Practices surrounded by a plain line are practices designed specifically for mycorrhiza. Practices not outlined contain only multipurpose proposals. This concept tree invites new practices, which are symbolized by the dashed lines

from clusters 4 and 5 almost exclusively focused on concept $\mathrm{C} 2$, while the farmers from cluster 3 focused on either concept $\mathrm{C} 1$ or $\mathrm{C} 3$. Although these farmers did not seem to integrate their proposals in a redesign approach, a majority ( 24 farmers of 27) proposed a practice mobilizing ecological interactions (C2 or C3), which places them at least in a substitution approach (Hill and MacRae 1995). These results support our first hypothesis that MYMYX leads farmers to internalize the fact that mycorrhization requires new methods outside the productionist paradigm.

Interestingly, the proportion of certified organic farms in the "proactive pool" was higher than that in the "reserved pool," with $69 \%$ and $22 \%$, respectively. It is likely that the constraints and development pathways associated with organic production certification lead farmers to integrate systemic considerations into the design of their farming systems (Padel 2008). This finding supports our second hypothesis that the sociotechnical context of farmers (participation in input reduction networks and organic certification) affects the outcome of MYMYX.

These results indicate the potential of MYMYX to address different populations and bring farmers one step further than their initial positions in the transition toward designing biodiversity-based agrosystems. 


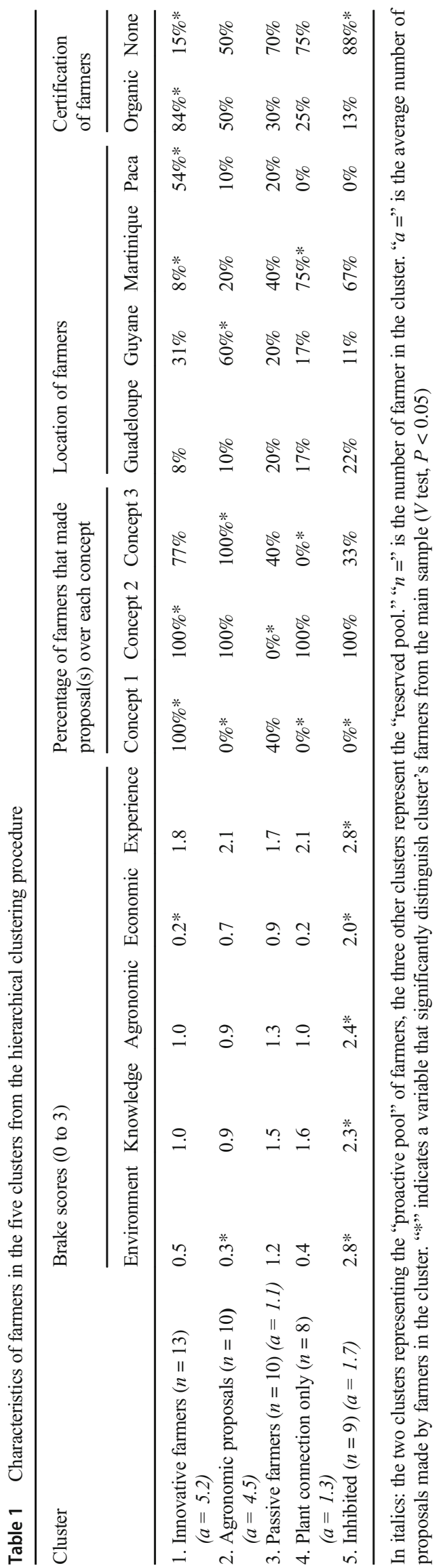

\subsection{Implementing a site-specific approach}

The type and diversity of practices proposed varied among the study areas (Fig. 4). Over the fourteen types of practices proposed, we observed higher diversity among Provence and French Guiana farmers, while the Guadeloupe and Martinique proposals were more focused on intercropping, rotation and fertilizer reduction. Here, we explore the influence of local sociotechnical context on proposal diversity.

In Provence (13 types of practices proposed), each practice aimed at reducing soil disturbance (limiting tillage, mulch, direct seeding) was proposed by more than $50 \%$ of farmers. Interestingly, the farmers also identified tillage reduction as their major technical constraint (on average 1.1 points higher than constraints to input reduction or increasing rotation). This result is in line with the current technical concerns of these Provence farmers, who are involved in the development of conservation agriculture to preserve and restore soils. Despite the constraints identified, the proposals were numerous and varied. Moreover, producing local propagules was also proposed by more than $50 \%$ of Provence farmers, which shows their advanced technical knowledge and their motivation to valorize indigenous resources.

In Martinique (seven types of practices proposed), more than $50 \%$ of the farmers involved in the study proposed practices focusing on both intercropping and rotation (C2). Secular know-how from the Creole garden explains this higher acquaintance with mixed cropping systems (Sinoquet and Cruz 1995). Farmers from Martinique were the only ones who proposed the possibility of collectively producing local strains to reduce input dependency while maintaining acceptable workload and investment levels. Extension network dynamics may also have played a role, as the surveyed farmers from Martinique had already worked together within the FREDON. In addition, they unanimously rejected the introduction of standard strains. Indeed, a recent sanitary crisis due to the use of persistent insecticide (chlordecone) created a climate of mistrust of imported inputs. Moreover, these farmers clearly expressed concerns about the introduction of potentially invasive or harmful pathogens that could damage the fragile insular biodiversity.

In Guadeloupe (seven types of practices proposed), more than $50 \%$ of farmers proposed intercropping, which may be partially inspired by the Creole garden, as in Martinique. However, this proposal type is also consistent with the limited opportunities for rotation in their monocropping sugarcane systems; intercropping is the only practice to mobilize C2 when rotation is excluded. Despite these constraints, Guadeloupe farmers came up with an original proposition. To overcome rotation issues for specialized farms, they imagined a cooperation between sugarcane farmers and specialized market gardeners to exchange plots and create a mutually beneficial rotation. Although this proposal would require an 


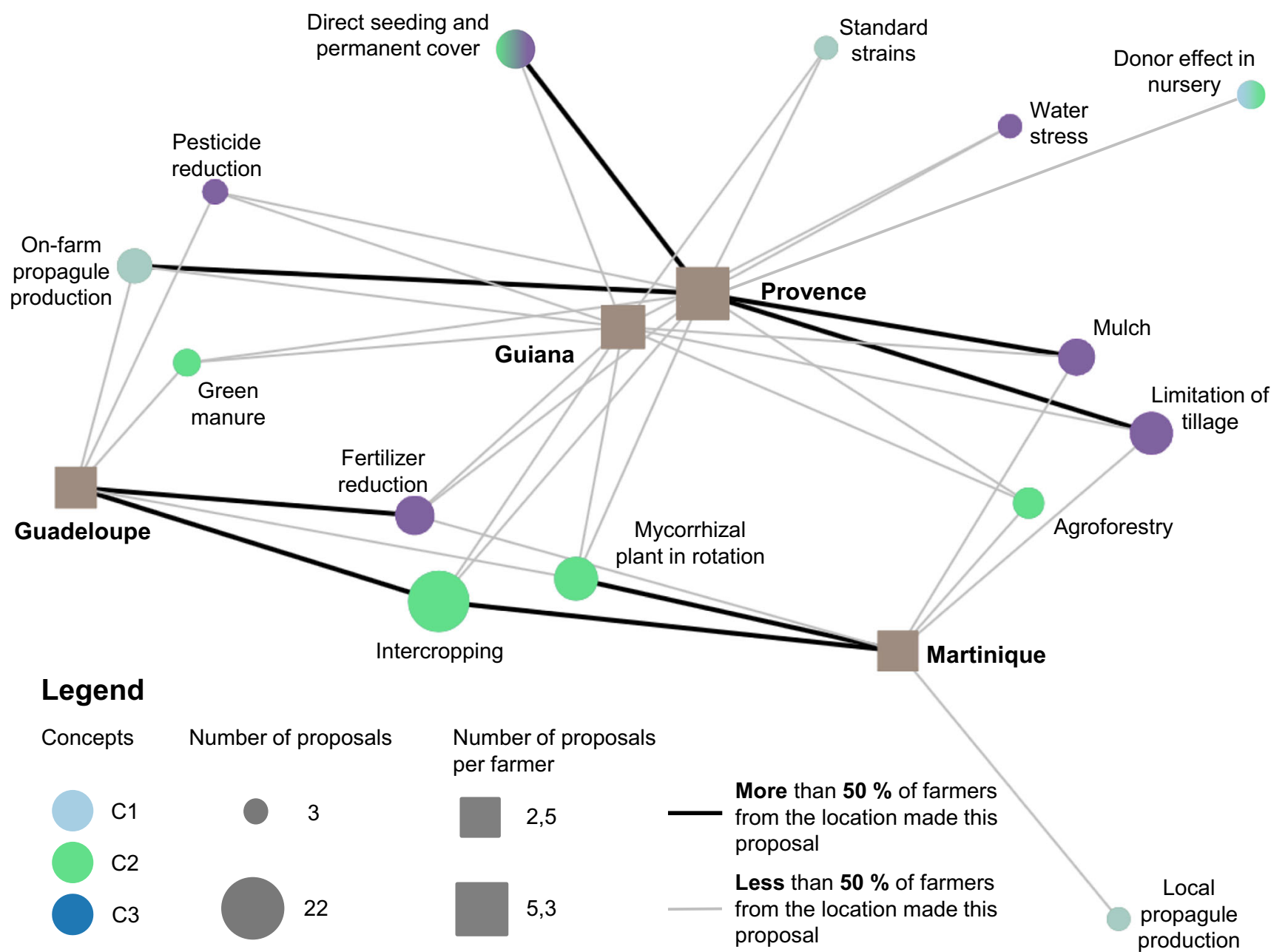

Fig. 4 Network of practices proposed per study area. Round nodes represent practices; their size is proportional to their frequency across all proposals. Square nodes represent locations; their size is

active investment from the farmers and extension services to be implemented, it shows the potential of farmers to think outside of their constraint frame and imagine solutions at a broader scale with local stakeholders. Moreover, more than $50 \%$ of farmers proposed fertilizer reduction. This proposal is compatible with their technical-group objective of reducing inputs, a crucial economic constraint for specialized sugarcane farms. As in Martinique, and certainly for the same reasons, Guadeloupe farmers rejected the introduction of standard propagules.

French Guiana's farmers proposed a wide diversity of practices (eleven), but no preferences stood out. As most of the French Guiana farmers are very isolated (due to lack of transport and communication infrastructure), they are accustomed to independently seeking and testing a diversity of solutions to their constraints (e.g., access to inputs). Notably, two-thirds of agroforestry proposals came from French Guiana (6 out of 9); this results from the experience of these farmers in the design and use of such systems, for example, with Inga trees (grain legume). proportional to the number of proposals per farmer. Black edges indicate that more than $50 \%$ of farmers from the connected location proposed the connected practice

In conclusion, we observed situational preferences for certain practices, which can be explained by the sociotechnical context, although local specificities may certainly be affected by the low sample size and the recruitment bias due to the involvement of professional networks. Nonetheless, MYMYX allowed farmers to adapt their proposals to their specific constraint frames, using scientific and vernacular knowledge.

\subsection{Leading an action-oriented approach}

Farmers identified the experience constraint (lack of local references and recognitions of practices) as the major constraint in the implementation of mycorrhiza-friendly agrosystems (all $P$ values $<0.01)$. They mentioned their reluctance to adopt several available practices due to lack of local evidence of effectiveness. To overcome this constraint, one-third of farmers (at the time of our survey) tested on their farms the solutions they had suggested. They also proposed to assess 
collectively some of the solutions they had identified. Discussions during the prospective workshop allowed farmers from two locations to initiate the design of collective experiments to screen, adjust, and test candidate solutions in local conditions.

The debate over the creation of a start-up producing selected local mycorrhizal strains for farmers allowed exploration of the practice of local production of propagules ( $\mathrm{C}$, see Fig. 3). In French Guiana, as in Martinique, some farmers were ready to buy such locally produced inputs, given an affordable price and a proven enhancement of biological soil activity. They insisted on the complementarity of this solution in association with other practices (mulch, compost, crop association, etc.). Other participants asked for on-farm propagule production. They argued that local strains would be more adapted to "their own soils and crops" and would present less risk of "natural disorder." They also stressed that "natural resources are free" and "farmers should be independent and self-reliant."

Following these debates, farmers asked for an experimental evaluation of different candidate mycorrhization practices involving indigenous or exogenous propagules. These demands have been supported and implemented by groups of local stakeholders involved in new projects that go beyond MYMYX. In Martinique, the FREDON, in collaboration with INRA, built an action-research project. In French Guiana, a group assembled a farmer cooperative, a technical institute, and the technical platform of an agricultural school.

Experiments involving farmers were performed in French Guiana to test the potential of candidate modalities to increase root colonization of cucumber (Cucumis sativus) by arbuscular mycorrhizal fungi in nurseries on three different farms (Chave and Angeon 2018). Three modalities and an additional control were assessed: donor effect of sorghum (Sorghum bicolor) grown in a tray for 8 weeks to cucumber seedlings (DE), inoculation of cucumber seedlings with indigenous strains grown on sorghum in a tray for 8 weeks (C1_in), inoculation of cucumber seedlings with commercial propagules (C1_ex). The control treatment consisted of sterilized and non-inoculated soil (-M). Root colonization of six cucumber plants per modality was evaluated according to Phillips and Hayman (1970). Values of frequency $(\% F)$ and intensity $(\% I)$ of root colonization by arbuscular mycorrhizal fungi were compared using Tukey's honestly significant difference test $(P \leq 0.05)$. Three weeks after sowing, on the three farms, the donor effect modality (DE) allowed for rapid and high mycorrhization of cucumber plants $(95.5 \pm 5.8 \% F$ and 32.6 $\pm 13.35 \% I$ on farm $1 ; 79.4 \pm 14.2 \% F$ and $8.5 \pm 4.5 \% I$ on farm 2 ; and $83.4 \pm 8.9 \% F$ and $11.6 \pm 2.6 \% I$ on farm 3 ) compared with the control treatment $-\mathrm{M}(5 \pm 3.5 \% \mathrm{~F}$ and 0 $\pm 0 \% I$ on farm $1 ; 1.1 \pm 1.7 \% F$ and $0 \pm 0 \% I$ on farm 2 ; and $1.7 \pm 2.8 \% F$ and $0 \pm 0 \% I$ on farm 3$)(P<0.05)$. The mycorrhization of cucumber seedlings of the donor effect modality (DE) showed significantly higher frequencies and intensities of root mycorrhization compared with $\mathrm{C} 1$ in modality on farms 1 and $3(75.5 \pm 16.4 \% F$ and $17.2 \pm 8.3 \% I$ on farm $1 ; 43.9 \pm 10.2 \% F$; and $3.1 \pm 3.7 \% I$ on farm 3$)(P<0.05)$ and significantly higher frequencies and intensities compared with $\mathrm{C} 1$ ex on farms 1 and $2(70.5 \pm 8.8 \% F$ and $6 \pm 3.6 \% I$ on farm $1 ; \overline{4} 9.5 \pm 9.8 \% F$ and $2 \pm 1.3 \% I$ on farm 2$)(P<0.05)$. These results indicate that valorization of indigenous mycorrhizal fungi strains was an efficient practice to increase root colonization of cucumber in the nursery. This finding is consistent with Pellegrino et al. (2011), who compared indigenous and standard inoculants. Thus, we showed, for the first time though on-farm experiments, that valorization of indigenous mycorrhizal fungi using a donor plant was an effective practice to increase root colonization by arbuscular mycorrhizal fungi before planting.

Such individual or collective assessment of candidate solutions helps to partially reduce the experience constraint. The pre-existing relations among the farmers certainly played a role in the fact that collective proposals emerged from only two study areas. Although the possibility of exchanging knowledge was appreciated by all farmers in MYMYX, the short duration of the workshop did not allow the building of new projects in all study areas. However, a sound extension approach could build upon MYMYX outcomes to engage farmers in a continuous improvement dynamic.

\subsection{Maintaining a continuous improvement dynamic}

MYMYX allowed farmers to link agricultural practices with the complex biological processes involved in mycorrhization, as represented by the concepts (Fig. 3). This development gave them new choices and the ability to explore new horizons to improve their farming systems by harnessing biodiversity. MYMYX also highlights the role of "boundary actors" (Tozik 2016) in carrying crucial information between a priori separated communities (scientists, farmers, councilors, extension services), facilitating communication and coordinating activities on agroecological concerns, bridging different agroecological networks. In doing so, they may facilitate agroecological technology acceptance by developing links between users or potential users. These boundary actors thus assume thus responsibility for agroecological technology transitionto-use and act as key actors that federate innovations. Boundary actors have been able to take over in the field to maintain the momentum created: training other farmers, accompanying them, and experimenting with new practices in two locations. They also ensured information dissemination on a larger scale through various tools: technical communications and advice and support for new farmers in these alternative approaches. In French Guiana, for example, the experiments described above (section 3.4) were the topics of a workshop of the Intertropical Agroecology Exchange Network that farmers not previously involved with MYMYX attended. Organized 
by the cooperative Bio Savane and the Chamber of Agriculture, it allowed the presentation of the farmers' results and visualization of mycorrhizae through the observation of roots under a microscope. Bio Savane then produced and disseminated a technical note on mycorrhizae. In Martinique, FREDON represented a major boundary actor, relaying the encouraging results of the previous experiments. It is the coordinator of a new long-term project coconstructed with most of the territory's technical partners: the agricultural chamber, an experimental station, an agricultural school, several farmers, and INRA. It aims to take experimentation to the next level by codesigning and evaluating complete cropping systems for the valorization of mycorrhizae and other key soil organisms.

In such multiactor approaches, favoring knowledge exchange is crucial. The MYMYX results were added to a knowledge sharing tool: the GECO web platform. This collaborative tool aims to share reliable and structured knowledge of agricultural practices among all stakeholders in agriculture. A form is intended to be completed by farmers on the basis of their own experiences and testimony of innovative agroecological practices. These claims agree with the proposals of Rillig et al. (2016), which advocated database creation and adaptation of plant breeding strategies to the local needs of farmers.

Although limited to small groups of farmers, the MYMYX approach has allowed (i) the appropriation of the mycorrhizal stakes by different stakeholders and (ii) the initiation and development of knowledge exchange tools. These results will likely support the establishment of new learning communities able to develop practices that are adapted to local constraints and challenges, which these communities could disseminate to the larger public.

\section{Conclusion}

In the context of commercial development of bioinoculants and facing the incompleteness of scientific- and practicalrelated knowledge, we showed that codesigning mycorrhizafriendly agrosystems promotes alternatives to mycorrhizal inoculants. We thus demonstrated that MYMYX, a methodological framework based on knowledge sharing, contributes to five key interrelated principles needed for the design of biodiversity-based agrosystems (Fig. 2). By proposing practices harnessing indigenous mycorrhizae through various current or innovative agronomic means, farmers could overcome the productionist paradigm, which had previously represented the only available option for them, placing them at least in a substitution approach (Hill and MacRae 1995).

At present, although mycorrhizae efficacy is controversial (Hart et al. 2018; Ryan and Graham 2018; Rillig et al. 2019; Ryan et al. 2019), most authors agree on the need for new methods to disentangle the impact of arbuscular mycorrhizal fungi in the field. Rillig et al. (2016) advocate to produce easyto-use tools for on-site mycorrhizal abundance and diversity monitoring, plant breeding programs prioritizing plant mycorrhizal responsiveness, and mycoengineering (promotion of mycorrhizal strains with desirable traits) while Ryan and Graham (2018) emphasize the need for comprehensive agronomic approaches. Our codesigning approach shows that a plurality of actors can contribute to knowledge production.

Our framework opened new avenues for cropping system ecologization. The sociotechnical environments of the farmers influenced their ability to integrate multiobjective and embedded solutions in a systemic approach. In that sense, MYMYX should be seen as one tool among many, useful to bring farmers one step further than they already are in their ability to innovate independently, engage and implement the agroecological transition.

Involving a larger agricultural community (technical institutes, cooperatives, high schools) has proven to be an interesting approach to start the assembly of learning communities. These outcomes of MYMYX were, however, dependent on the motivation and interest of local stakeholders, as well as their sociotechnical context. In that sense, the development of marketing campaigns oriented toward standard bioinoculants could increase interest and questioning about mycorrhizae. As with many participative approaches, we show that boundary actors are crucial to support and feed innovation dynamics. The implementation of MYMYX calls for an extension-wide effort to involve initial learning actors along with technical institutes to promote development approaches following the five principles.

More generally, by raising the issue of mycorrhizae, MYMYX tackles the complexity and uncertainty of ecological interactions. This type of approach can assist farmers in biodiversity-based agrosystem design and could be employed to discuss other ecological interactions.

Acknowledgments The authors would like to thank Amélie Lefèvre, Jean-Louis Diman, Arnaud Dufils, and Arnaud Larade and the technical teams of the INRA units Alénya, Peyi and ASTRO for their contributions to the workshops, Anne-Charlotte Harter for the experiments, and Mireille Navarrete for her comments on the manuscript.

Funding information This research was part of the SYSTEMYC project funded by l'Agence Française pour la Biodiversité (call for project: "Pour et sur le plan EcoPhyto"). It was also financially supported by the Sustainable Management of Crop Health INRA program and the Labex CEBA (Centre d'Étude de la Biodiversité Amazonienne).

Data availability The datasets generated during and analyzed during the current study are not publicly available but are available from the corresponding author on reasonable request.

\section{Compliance with ethical standards}

Conflict of interest The authors declare that they have no conflict of interest. 
Open Access This article is distributed under the terms of the Creative Commons Attribution 4.0 International License (http:// creativecommons.org/licenses/by/4.0/), which permits unrestricted use, distribution, and reproduction in any medium, provided you give appropriate credit to the original author(s) and the source, provide a link to the Creative Commons license, and indicate if changes were made.

\section{References}

Augé RM (2001) Water relations, drought and vesicular-arbuscular mycorrhizal symbiosis. Mycorrhiza 11:3-42. https://doi.org/10.1007/ s005720100097

Bender SF, Wagg C, van der Heijden MGA (2016) An underground revolution: biodiversity and soil ecological engineering for agricultural sustainability. Trends Ecol Evol 31:440-452. https://doi.org/ 10.1016/j.tree.2016.02.016

Buckwell A, Nordang Uhre A, Williams A et al (2014) Sustainable intensification of European agriculture. Institute for European Environmental Policy, Brussels http://www.risefoundation.eu/ images/files/2014/2014_\%20SI_RISE_FULL_EN.pdf

Chave M, Tchamitchian M, Ozier-Lafontaine H (2014) Agroecological engineering to biocontrol soil pests for crop health. In: Sustainable agriculture reviews 14: agroecology and global change. Springer, pp 269-297. https://doi.org/10.1007/978-3-319-06016-3 8

Chave M, Angeon V (2018) Du partage de connaissances à la coconception d'innovations agroécologiques : Exemple de la mobilisation des mycorhizes en Guyane. Innov Agron 64:97-111 https:/www6.inra.fr/ciag/content/download/6363/46619/file/ Vol64-9-Chave.pdf

Douds DD, Nagahashi G, Pfeffer PE et al (2005) On-farm production and utilization of arbuscular mycorrhizal fungus inoculum. Can J Plant Sci 85:15-21. https://doi.org/10.4141/P03-168

Duru M, Therond O, Martin G et al (2015) How to implement biodiversity-based agriculture to enhance ecosystem services: a review. Agron Sustain Dev 35:1259-1281. https://doi.org/10.1007/ s13593-015-0306-1

Hart MM, Antunes PM, Chaudhary VB, Abbott LK (2018) Fungal inoculants in the field: Is the reward greater than the risk? Funct Ecol 32: 126-135. https://doi.org/10.1111/1365-2435.12976

Hill SB, MacRae RJ (1995) Conceptual framework for the transition from conventional to sustainable agriculture. J Sustain Agric 7:81-87. https://doi.org/10.1300/J064v07n01 07

Jansa J, Wiemken A, Frossard E (2006) The effects of agricultural practices on arbuscular mycorrhizal fungi. J Geol Soc Lond 266:89-115. https://doi.org/10.1144/gsl.sp.2006.266.01.08

Johnson D, Gilbert L (2015) Interplant signaling through hyphal networks. New Phytol 205:1448-1453. https://doi.org/10.1111/nph. 13115

Le Masson P, Hatchuel A, Weil B (2009) Design theory and collective creativity: a theoretical framework to evaluate KCP process. In: Proceedings of the 17th International Conference on Engineering Design. Palo Alto, vol 6, pp 277-288 https://www.designsociety. org/publication $/ 28745 /$ Design+Theory+and+Collective+ Creativity\%3A+A+Theoretical+Framework+to+Evaluate+KCP+ Process

Martin G (2015) A conceptual framework to support adaptation of farming systems - development and application with forage rummy. Agric Syst 132:52-61. https://doi.org/10.1016/j.agsy.2014.08.013

Méndez EV, Bacon CM, Cohen R (2013) Agroecology as a transdisciplinary, participatory, and action-oriented approach. Agroecol Sust Food 37:3-18. https://doi.org/10.1080/21683565.2017.1358962
Meynard J-M, Dedieu B, Bos AP (2012) Re-design and co-design of farming systems. An overview of methods and practices. In: Farming systems research into the 21 st century: the new dynamic. Springer Netherlands, Dordrecht, pp 405-429. https://doi.org/10. 1007/978-94-007-4503-2 18

Padel S (2008) Values of organic producers converting at different times: results of a focus group study in five European countries. Int J Agric Resour Gov Ecol 7:63. https://doi.org/10.1504/IJARGE.2008. 016980

Pellegrino E, Bedini S, Avio L et al (2011) Field inoculation effectiveness of native and exotic arbuscular mycorrhizal fungi in a Mediterranean agricultural soil. Soil Biol Biochem 43:367-376. https://doi.org/10. 1016/j.soilbio.2010.11.002

Phillips JM, Hayman DS (1970) Improved procedures for clearing roots and straining parasitic and vesicular-arbuscular mycorrhizal fungi for rapid assessment of infection. J Brit Mycol Society 55:158161. https://doi.org/10.1016/s0007-1536(70)80110-3

R Core Team (2018) R: A Language and Environment for Statistical Computing. R Foundation for Statistical Computing, Vienna. https://www.R-project.org

Rillig MC, Sosa-hernández MA, Roy J (2016) Towards an integrated mycorrhizal technology : harnessing mycorrhiza for sustainable intensification in agriculture. Front Plant Sci 7:1-5. https://doi.org/10. 3389/fpls.2016.01625

Rillig MC, Aguilar-Trigueros CA, Camenzind Tet al (2019) Why farmers should manage the arbuscular mycorrhizal symbiosis. New Phytol 222:1171-1175. https://doi.org/10.1111/nph.15602

Ryan MH, Graham JH (2018) Little evidence that farmers should consider abundance or diversity of arbuscular mycorrhizal fungi when managing crops. New Phytol 220:1092-1107. https://doi.org/10. 1111/nph.15308

Ryan MH, Graham JH, Morton JB, Kirkegaard JA (2019) Research must use a systems agronomy approach if management of the arbuscular mycorrhizal symbiosis is to contribute to sustainable intensification. New Phytol 222:1176-1178. https://doi.org/10.1111/nph.15600

Salembier C, Segrestin B, Berthet E et al (2018) Genealogy of design reasoning in agronomy: Lessons for supporting the design of agricultural systems. Agric Syst 164:277-290. https://doi.org/10.1016/j. agsy.2018.05.005

Sinoquet and Cruz (1995) Ecophysiology of tropical intercropping. INRA Editions, Paris

Smith SE, Read DJ (2008) Mycorrhizal symbiosis, 3rd edn. Academic Press, London

Star SL, Griesemer JR (1989) Institutional ecology, translations' and boundary objects: amateurs and professionals in Berkeley's Museum of Vertebrate Zoology. Soc Stud Sci 19:387-420. https:// doi.org/10.1177/030631289019003001

Tozik S (2016) Actor-networks and boundary actors in capability integration theory. In: 26th Annual INCOSE International Symposium, Edinburgh

Verbruggen E, van der Heijden MGA, Rillig MC, Kiers ET (2013) Mycorrhizal fungal establishment in agricultural soils: factors determining inoculation success. New Phytol 197:1104-1109. https:// doi.org/10.1111/j.1469-8137.2012.04348.x

Voets L, de la Providencia IE, Fernandez K et al (2009) Extraradical mycelium network of arbuscular mycorrhizal fungi allows fast colonization of seedlings under in vitro conditions. Mycorrhiza 19: 347-356. https://doi.org/10.1007/s00572-009-0233-6

Wezel A, Soldat V (2009) A quantitative and qualitative historical analysis of the scientific discipline of agroecology. Int J Agric Sustain 7: 3-18. https://doi.org/10.3763/ijas.2009.0400

Publisher's note Springer Nature remains neutral with regard to jurisdictional claims in published maps and institutional affiliations. 